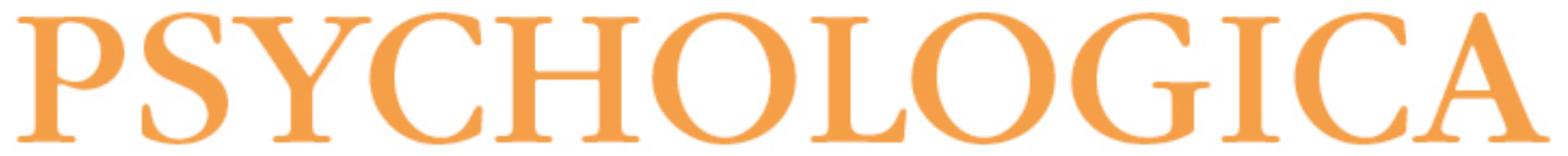

\title{
Avaliação da percepção da aceitação da família e dos amigos: contributos para a validade da estrutura factorial da Perceived Aceptance Scale (PAS)
}

Autor(es): $\quad$ Figueiredo, Cláudia; Maia, José; Pinheiro, Maria do Rosário

Publicado por: Imprensa da Universidade de Coimbra

URL

persistente: URI:http://hdl.handle.net/10316.2/5527

DOI: DOI:http://dx.doi.org/10.14195/1647-8606_53_13

Accessed : $\quad$ 26-Apr-2023 10:16:22

A navegação consulta e descarregamento dos títulos inseridos nas Bibliotecas Digitais UC Digitalis, UC Pombalina e UC Impactum, pressupõem a aceitação plena e sem reservas dos Termos e Condições de Uso destas Bibliotecas Digitais, disponíveis em https://digitalis.uc.pt/pt-pt/termos.

Conforme exposto nos referidos Termos e Condições de Uso, o descarregamento de títulos de acesso restrito requer uma licença válida de autorização devendo o utilizador aceder ao(s) documento(s) a partir de um endereço de IP da instituição detentora da supramencionada licença.

Ao utilizador é apenas permitido o descarregamento para uso pessoal, pelo que o emprego do(s) título(s) descarregado(s) para outro fim, designadamente comercial, carece de autorização do respetivo autor ou editor da obra.

Na medida em que todas as obras da UC Digitalis se encontram protegidas pelo Código do Direito de Autor e Direitos Conexos e demais legislação aplicável, toda a cópia, parcial ou total, deste documento, nos casos em que é legalmente admitida, deverá conter ou fazer-se acompanhar por este aviso. 
NÚMERO 53

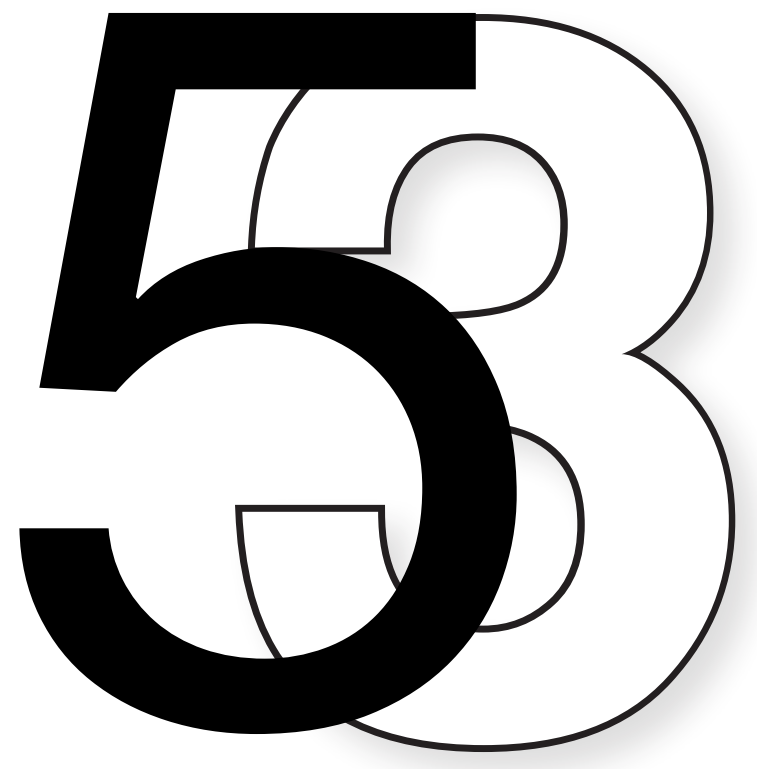

IMPRENSA DA UNIVERSIDADE DE COIMBRA

FACULDADE DE PSICOLOGIA E DE CIÊNCIAS DA EDUCAÇÃO DA UNIVERSIDADE DE COIMBRA 


\section{Avaliação da percepção da aceitação da família e dos amigos: contributos para a validade da estrutura factorial da Perceived Aceptance Scale (PAS)}

Cláudia Figueiredo', José Maia² \& Maria do Rosário Pinheiro³

A Escala de Percepção da Aceitação-PAS (Brock, Sarason, Sanghvi \& Gurung, 1998; Versão portuguesa de Pinheiro \& Ferreira, 2001) mede, nos relacionamentos com o pai, a mãe, a família e os amigos, a apreciação pessoal de que os outros se preocupam connosco e nos valorizam independentemente das nossas atitudes e acções pontuais.

Procurando contribuir para o processo de validação transcultural da PAS neste estudo, a partir de uma amostra de estudantes universitários, são apresentados e discutidos os resultados da Análise Factorial Confirmatória com recurso a um estimador apropriado para lidar com dados ordinais (Muthén \& Muthén, 2005).

Conclui-se que os modelos propostos reproduzem adequadamente a estrutura de cada subescala e da escala no seu todo. Na análise do ajustamento local verifica-se que os valores de $R^{2}$ que cada factor extrai por item se situam entre .11 e .8o, requerendo uma interpretação cuidada da qualidade semântica de alguns deles. São sugeridas as respectivas alterações.

PALAVRAS-CHAVE: Suporte social, Psicometria, Percepção da aceitação, Análise factorial confirmatória

\section{Introdução}

A Escala de Percepção da Aceitação (PAS), construída por Brock, Sarason, Sanghvi e Gurung, (1998), surge no intuito de operacionalizar a variável sentido da aceitação (sense of acceptance) proposta por Sarason, Pierce e Sarason (1990), no âmbito do Modelo Transaccional do Suporte Social. De acordo com este quadro conceptual, o sentido da aceitação assume um papel central na leitura da disponibilidade das

1 Doutoranda da Faculdade de Psicologia e de Ciências da Educação da Universidade de Coimbra claudiarcfigueiredo@gmail.com

2 Professor Catedrático da Faculdade de Ciências do Desporto e Educação Física da Universidade do Porto

3 Professora Auxiliar da Faculdade de Psicologia e de Ciências da Educação da Universidade de Coimbra 
entidades de suporte e, desta forma, no ajustamento psicológico dos indivíduos (Brock et al, 1998, Sarason, Pierce \& Sarason, 1990; Pinheiro \& Ferreira, 2001, Figueiredo, 2005, Figueiredo, Maia \& Pinheiro, 2004, 2005).

A PAS representa uma proposta de delimitação do sentido da aceitação definindo a percepção da aceitação como uma característica relativamente estável da personalidade, relativa a relacionamentos concretos com os amigos, a mãe, o pai e a família, e descrita como uma avaliação de que "os outros se preocupam connosco e nos valorizam e que esse cuidado não é dependente de por vezes as nossas atitudes e acções serem diferentes das habituais" (Brock et al., 1998, p.6). Esta operacionalização constitui uma ferramenta com importantes potencialidades, quer no âmbito da investigação com estudantes do ensino superior, quer no próprio trabalho de intervenção dos profissionais responsáveis pelo aconselhamento e apoio a este público. Esta ideia é fortalecida pelos resultados das investigações, na medida em que níveis mais elevados de percepção da aceitação se apresentaram associados a niveis superiores de vivências adaptativas, tanto ao nível académico e vocacional, como pessoal e social (Pinheiro, 2003; Turner, Sarason \& Sarason, 2001).

Num momento tão particular da vida dos estudantes como o período de frequência universitária será necessário que estes sintam, por um lado, os novos colegas e professores como disponíveis para os ouvir, apoiar e encorajar e, por outro lado, a segurança de que os outros significativos se disponibilizarão a acolhe-los mesmo que esta seja uma fase de exploração, longe das pessoas e do ambiente de origem. Para este sentimento de segurança e confiança em si próprio é fundamental que se percebam aceites, nas suas características próprias e individuais.

No contexto do ensino superior, quando vários estabelecimentos têm promovido a criação de serviços de apoio aos estudantes, e atendendo à importância que a informação acerca desta variável pode representar, parece adequado disponibilizar, mesmo que indirectamente, um instrumento de medida que possibilite aos profissionais, que trabalham com esta população, a oportunidade de procederem a intervenções devidamente fundamentadas. Estando a Escala de Percepção da Aceitação, a PAS (Perceived Acceptance Scale), traduzida em língua portuguesa e oferecendo evidências da sua viabilidade tanto no domínio da investigação como na intervenção, o passo que, neste momento, se anunciava como imperioso seria o de aprofundar a análise das suas qualidades psicométricas essencialmente nos aspectos da fidelidade e da validade ${ }^{4}$.

4 O trabalho que se apresenta é parte integrante da investigação descrita na dissertação de mestrado, intitulada "Percepção da Aceitação: estudo de validade de uma escala" (Figueiredo, 2005), apresentada à Faculdade de Psicologia e de Ciências da Educação da Universidade de Coimbra, sob a orientação dos segundos autores do presente artigo. 
Considerando "toda a escala psicológica é, em si mesma, uma possibilidade, entre várias, de descrição e representação de um dado conteúdo psicológico, (...) um modelo, um mapa imperfeito, o menos possível, de um aspecto representacional do sujeito" (Maia, 1996, p.44), foi objectivo deste trabalho explorar os diferentes aspectos desta possibilidade avançada para a avaliação da percepção da aceitação, com estudantes universitários.

\section{Método}

\subsection{Sujeitos}

A amostra é constituída por 1290 estudantes das oito Faculdades que constituem a Universidade de Coimbra, com idades compreendidas entre os 17 e os 44 anos de idade, com uma média de 21.27 anos ( $D P=2.96)$. A maioria destes indivíduos é de nacionalidade portuguesa ( $n=1210 ; 93.8 \%$ ) e estado civil solteiro ( $n=1224 ; 94.9 \%)$.

Na distribuição por género, 509 estudantes são do género masculino (39.5\%) e 781 do género feminino (60.5\%) (cf. Quadro 1).

Quuadro 1: Distribuição da amostra por género.

\begin{tabular}{lcc}
\hline Género & N & $\%$ \\
\hline Masculino & 509 & 39.5 \\
Feminino & 781 & 60.5 \\
Total & 1290 & 100.0 \\
\hline
\end{tabular}

Estes estudantes são na sua maioria primeiranistas ( $n=386 ; 29.9 \%)$ e terceiranistas $(n=348 ; 27 \%)$, sendo os $5^{\circ}$ e $6^{\circ}$ anos os menos representados nesta amostra (respectivamente, 3.1 e 1.2\%) (cf. Quadro 2). 
Quadro 2: Distribuição da amostra por ano de frequência.

278

\begin{tabular}{lcc}
\hline Ano de frequência & N & $\%$ \\
\hline $1^{\circ}$ ano & 386 & 29.9 \\
$2^{\circ}$ ano & 232 & 18.0 \\
$3^{\circ}$ ano & 348 & 27.0 \\
$4^{\circ}$ ano & 268 & 20.8 \\
$5^{\circ}$ ano & 40 & 3.1 \\
$6^{\circ}$ ano & 16 & 1.2 \\
Total & 1290 & 100.0 \\
\hline
\end{tabular}

\subsection{Instrumento}

A PAS (Brock et al., 1998) é um instrumento de auto-resposta composto por 44 itens respondidos numa escala de 5 pontos do tipo likert, desde (1) discordo fortemente a (5) concordo fortemente. Estes itens correspondem a uma apreciação acerca da aceitação de quatro entidades de suporte social: a mãe, o pai, os amigos e a família. A percepção da aceitação da mãe e do pai é avaliada através de 10 itens ( 6 positivos e 4 negativos) cuja construção semântica é igual nas duas subescalas. As medidas dos amigos e da família são concretizadas através de 12 itens em cada ( 6 positivos e 6 negativos), de conteúdos equivalentes.

Os estudos de tradução e adaptação para a população portuguesa conduzidos por Pinheiro e Ferreira (2001) foram efectuados com amostras de estudantes da Universidade de Coimbra. Nestes trabalhos, a Escala de Percepção da Aceitação revelou níveis de consistência interna satisfatórios, isto é, alfas de Cronbach a variar entre .88 (PAS Amigos e PAS Família) e .89 (PAS Mãe e PAS Família), assim como resultados de validade concorrente semelhantes aos encontrados pelos autores originais (Pinheiro, 2003).

Nas Análises em Componentes Principais realizadas quer pelos autores originais, quer pelos autores portugueses, as quatro subescalas foram estudadas isoladamente (Figueiredo, Maia \& Pinheiro, 2004; Pinheiro, 2003). Os resultados, de um modo geral, apontaram para a existência de uma só componente que explica as percepções da aceitação da mãe e do pai, enquanto nas subescalas dos amigos e da família foram extraídas duas componentes, uma referente aos itens que descrevem atitudes e sentimentos de aceitação e a outra referente aos aspectos que se podem interpretar como de rejeição. 


\subsection{Estratégias de investigação}

No intuito de concretizar os objectivos propostos e realizar um estudo que possibilite uma melhor compreensão acerca das características da PAS optou-se por um conjunto de procedimentos que se passa a explicitar.

A demonstração da adequação de um construto pelo teste é realizada com base amplamente utilizado na literatura (DeVellis, 2003; Thompson, 2003) e, neste caso específico, ter sido a opção nos estudos anteriores, o que facilita a comparação.

O cálculo do alfa de Cronbach é efectuado com base na média das intercorrelações entre todos os itens da escala, o que permite avaliar o grau de homogeneidade existente entre as respostas aos itens dentro de cada dimensão (Cronbach, 1984; Nunnally, 1978), partindo do pressuposto que cada questão terá um peso idêntico na marcação do factor.

Ainda dentro dos aspectos da consistência interna, na avaliação da qualidade dos itens recorreu-se a correlação de cada item com o total da própria subescala, excluindo o próprio item para que o valor não fosse inflacionado. Este procedimento apresenta a desvantagem de pressupor que todos os itens são somáveis e representam uma parte da variável latente (Pasquali, 2003), que no caso específico da PAS não será um aspecto tão preocupante uma vez que este instrumento já estava construído e tinha sido amplamente aplicado.

Num segundo momento, construiu-se um modelo explicativo dos dados com vista a proceder-se à Análise Factorial Confirmatória. Esta metodologia, inserida no âmbito dos modelos de equações estruturais, pressupõe a especificação de hipóteses que constituem o modelo a testar, bem como uma representação gráfica destas especificações. Assim, para cumprir o propósito de formular um modelo estatístico confiável acerca da forma como os indicadores medem a variável latente estes modelos colocam os investigadores perante o enorme desafio de traduzir a teoria num conjunto de equações estruturais e, seguidamente, testar em que medida o modelo formulado é um bom esquema de leitura para os dados observados (Muthén, 1992).

Na Análise Factorial Confirmatória é necessário atender a algumas etapas com vista a uma tomada de posição quanto ao modelo em teste. Na primeira etapa, constrói-se, de forma fundamentada, o modelo em análise. Esta é uma questão essencial numa metodologia que coloca em teste as concepções explicativas dos 
conceitos e das suas relações. Trata-se de testar os pressupostos teóricos subjacentes aos construtos, de perceber se o tipo de relações entre as variáveis que se tem subentendido, ou se as conclusões que se têm avançado, são efectivamente sustentados pelos dados recolhidos no terreno.

Na segunda etapa, considera-se a avaliação do ajustamento global do modelo aos dados, analisando se o modelo devidamente fundamentado na teoria se afasta, ou aproxima, da configuração da matriz de dados construída com os dados recolhidos. Para este efeito, na modelação de estruturas de covariância o teste formal ao modelo é realizado através do teste de qui-quadrado $\left(x^{2}\right)$, cujo valor se pretende que seja o mais pequeno possível para que se possa aceitar a hipótese subjacente ao teste e não rejeitá-la. Para além desta estatística e com a finalidade de apoiar a decisão quanto à adequação do modelo encontram-se à disposição dos investigadores diferentes tipos de indicadores de ajustamento global. No presente estudo optou-se pelo Comparative Fit Index (CFI), o Tucker and Lewis Index (TLI) e o Root Mean Square Error of Aproximation (RMSEA) por se considerar que estas medidas, dentro de um conjunto bastante extenso, seriam as mais apropriadas ao tipo de dados e de análise realizadas (Maia, 1996; Schermelleh-Engel, Moosbrugger \& Müller, 2003; Brown, 2006). O valor do teste de qui-quadrado $\left(x^{2}\right)$ remete para a noção de a matriz ajustada não ser significativamente diferente da matriz dos dados observados (Maia, 1996), logo um modelo adequado aos dados recolhidos que deve permitir a aceitação da hipótese nula (não existência de diferenças entre a duas matrizes). Contudo, este teste estatístico apresenta como limitação ser sensível a desvios à normalidade e linearidade e ao tamanho da amostra o que impõe que sejam utilizados em simultâneo outros indicadores (Schermelleh-Engel, Moosbrugger \& Müller, 2003).

O Comparative Fit Index (CFI) é uma estatística construída com base no teste de qui-quadrado e fornece uma indicação acerca da qualidade do modelo estimado relativamente ao modelo nulo, ou seja, reflecte o ganho substantivo no modelo com a imposição de estrutura. Os valores de corte seguidos para esta medida são .97 , indicador de bom ajustamento e .95, valor acima do qual se pode afirmar que o modelo é aceitável (Schermelleh-Engel, Moosbrugger \& Müller, 2003).

O Tucker and Lewis Index (TLI) é em tudo semelhante ao Comparative Fit Index (CFI), apresentando a enorme vantagem de não depender da dimensão amostral. Este índice desenvolvido inicialmente no contexto da Análise Factorial foi rapidamente incorporado na metodologia de Análise de Estruturas de Covariância devido às suas qualidades e tem sido amplamente reportado. O ponto de corte assumido para esta media situa-se em pelo menos .95 (Brown, 2006). 
Contudo, Brown (2006) refere que para ambos os indicadores se possam aceitar valores entre .90 e .95 , desde que os restantes aspectos em avaliação reforcem a aceitação do modelo.

O Root Mean Square Error of Aproximation (RMSEA) permite aferir da distância a que se encontra a matriz de covariância do modelo teórico e a matriz constituída com base nos dados recolhidos, ou seja, a extensão de erro entre uma hipótese nula que representaria uma excelente aproximação e os dados que efectivamente se possuem. O RMSEA é uma medida bastante referenciada na literatura uma vez que é relativamente independente do tamanho da amostra e beneficia a parcimónia do modelo. Na avaliação dos valores deste indicador têm sido sugeridos os seguintes valores de corte: (1) entre o e.05 para um bom ajustamento, (2) entre .05 e .08 para um ajustamento adequado e (3) entre .08 e .10 para um ajustamento medíocre (Schermelleh-Engel, Moosbrugger \& Müller, 2003).

Na terceira etapa, será necessário analisar os coeficientes gerados pelo modelo, ou seja, verificar se os parâmetros estimados de forma óptima são substantivamente interpretáveis atendendo às relações entre as variáveis. Assim, o ajustamento local é realizado pela análise dos valores dos coeficientes estandardizados e de $R^{2}$. A apreciação das saturações factoriais (loadings) justifica-se por ser importante atribuir significado à sua magnitude e sinal, esperando que estas estejam em consonância com o definido previamente no modelo semântico original. Espera-se que todos os loadings sejam significativamente diferentes de zero. A observação do valor de $R^{2}$ permite a reflexão acerca da importância de cada item para o factor. No entanto, no caso dos dados observados não serem contínuos, a literatura tem sugerido alguma precaução na interpretação deste valor como a percentagem de variância do item explicada pelo factor (Muthén \& Muthén, 2005).

Na análise da estrutura factorial subjacente à PAS procedeu-se a uma Análise Factorial Confirmatória considerando que os dados observados possuíam um nível de mensuração ordinal marcando uma variável latente contínua. Quando nos encontramos perante este pressuposto é fortemente sugerido, embora nem sempre se seguia esta sugestão, a utilização de uma estratégia analítica que use o estimador WLSMV (Weighted least square parameter estimates using a diagonal weight matrix with robust standard errors and means), tal como indicado pelo trabalho pioneiro de Bengt Muhén e implementado no programa Mplus (Muthén \& Muthén, 2005). Este estimador sendo o mais adequado, acarreta o inconveniente de a maioria dos pontos de corte e intervalos de confiança, sugeridos na literatura, para os indicadores de ajustamento global do modelo se referirem ao estimador mais amplamente utilizado, o estimador de máxima verosimilhança (Flora \& Curran, 2004). Para ultrapassar este problema optou-se por seguir a proposta avançada 
pelos autores do software, sendo que no caso do RMSEA não são disponibilizados os valores do intervalo de confiança, apenas a estimativa pontual.

\subsection{Procedimentos}

A amostra foi recolhida nas oito faculdades da Universidade de Coimbra, tendo o preenchimento dos questionários decorrido em diferentes locais frequentados pelos estudantes. Para além das salas de aula, recorreu-se ainda a bibliotecas, salas de leitura e estudo e espaços de convivio. Esta alternância dos locais de recolha pretendeu tornar a amostra mais diversificada e heterogénea relativamente a diferentes características como serem estudantes que não frequentam habitualmente as aulas ou que utilizam os espaços da universidade para estudar. A participação, através do preenchimento do questionário, foi voluntária e solicitada após o esclarecimento do âmbito e objectivos da investigação, assim como do tempo de duração da tarefa.

No tratamento estatístico dos dados recorreu-se aos programas SPSS 15.0 e Mplus 3 (Muthén \& Muthén, 2005).

\section{Apresentação e discussão de resultados}

\subsection{Caracteristicas psicométricas}

O estudo psicométrico da PAS incidirá nas quatro subescalas que compõem esta escala, considerando um ou dois factores para a sua explicação, uma vez que serão testadas diferentes configurações (para as quatro entidades separadamente e para o modelo global).

Assim, a avaliação da qualidade dos itens da Percepção da Aceitação da Mãe (PAS Mãe) revela índices de correlação com o score total da subescala a variar entre .53 e .72 , nos itens 30 e 18 , respectivamente. Sendo estas correlações superiores a .30, critério apontado por Cronbach (1984) para a retenção de itens. O alfa de Cronbach para a PAS Mãe apresentou o valor .88 (cf. Quadro 3).

Os itens da Percepção da Aceitação do Pai (PAS Pai) apresentam coeficientes de correlação entre .42 (item 4) e .77 (item 24), o que revela valores satisfatórios de relevância dos itens para o total da subescala. O índice de consistência interna possui o valor .90 (cf. Quadro 3). 
As correlações dos itens da Percepção da Aceitação dos Amigos (PAS Amigos) com o score da subescala situam-se entre .32 (item 25) e .63 (item 21). Estes valores apontam no sentido da adequação das questões colocadas para medir esta variável. O alfa de Cronbach foi de .85 , indicativo de uma boa consistência interna (cf. Quadro 3).

Na análise dos itens da PAS Amigos foi também considerada a separação em duas subescalas, referentes aos aspectos de aceitação ou de rejeição, tal como tinha sido referido pelos autores que realizaram investigações com esta escala anteriormente (Brock et al., 1998; Pinheiro, 2003) e porque esta proposta será submetida a Análise Factorial Confirmatória. A observação dos novos valores de correlação do item com o total da subescala fornece, uma vez mais, a informação de que as correlações mais baixas se referem aos itens 25 e 43 (respectivamente, .38 e .36), sendo, porém, todos superiores a .30. O alfa de Cronbach da dimensão aceitação situou-se em .78, enquanto o da dimensão rejeição em .79 (cf. Quadro 3). Na análise psicométrica da Percepção da Aceitação da Família (PAS Família) seguiram-se os procedimentos referidos para a PAS Amigos. Esta opção justifica-se por dois motivos: o facto destas duas medidas terem configurações semânticas semelhantes traduzidas numa correspondência item a item e porque os procedimentos efectuados posteriormente serão idênticos.

Considerando a totalidade dos itens da PAS Família num único factor, os coeficientes de correlação e o alfa de Cronbach sugerem níveis confiáveis de consistência interna (cf. Quadro 3). O item que revela a correlação mais expressiva é o 23 a presentando um valor de .65. A questões 3 possuí a correlação mais baixa com total da subescala (.37). O valor obtido para o alfa de Cronbach é de .87.

$\mathrm{Na}$ análise alternativa considerando uma subescala de aceitação familiar e outra de rejeição familiar, verifica-se que todos as correlações dos itens com o score da subescala se situam acima de 30 , contudo os itens 35 e 3 revelam valores próximos desse ponto (respectivamente .33 e .35). O valor de alfa de Cronbach nos dois factores foi de 79 (cf. Quadro 3).

Quadro 3: Alfas de Cronbach $(\alpha)$ para as dimensões da PAS.

\begin{tabular}{llcc}
\hline Variáveis & Dimensões & N itens & $\alpha$ \\
\hline PAS Mãe & PAS Mãe & 10 & .88 \\
PAS Pai & PAS Pai & 10 & .90 \\
& PAS Amigos & 12 & .85 \\
\multirow{3}{*}{ PAS Amigos } & PAS Amigos Aceitação & 6 & .78 \\
& PAS Amigos Rejeição & 6 & .79 \\
& PAS Família & 12 & .87 \\
& PAS Família Aceitação & 6 & .79 \\
& PAS Família Rejeição & 6 & .79
\end{tabular}


Em síntese, todas as subescalas apresentam índices de consistência interna muito satisfatórios, tendo os estudos anteriores relatados valores semelhantes (Brock et al., 1998; Pinheiro, 2003).

\subsection{Estudo de validade da Escala de Percepção da Aceitação (PAS)}

Sendo a estrutura semântica dos itens da Escala de Percepção da Aceitação igual nas subescalas relativas ao pai e à mãe, assim como, entre as subescalas dos amigos e da família, nos estudos anteriores os autores optaram por proceder a quatro Análises em Componentes Principais, uma para cada uma das entidades de suporte avaliada (Brock et al., 1998; Pinheiro, 2003).

Neste trabalho considerou-se enriquecedor analisar as duas hipóteses, por um lado, construir quatro modelos interpretativos de cada subescala da PAS, por outro, construir um modelo composto por quatro subescalas, como variáveis latentes correlacionadas.

\subsubsection{Percepção da Aceitação da Mãe e do Pai}

Os modelos que avaliam a configuração factorial da PAS Mãe e da PAS Pai, separadamente são em tudo idênticos e compostos por 10 itens que marcam um único factor (cf. Figura 1).
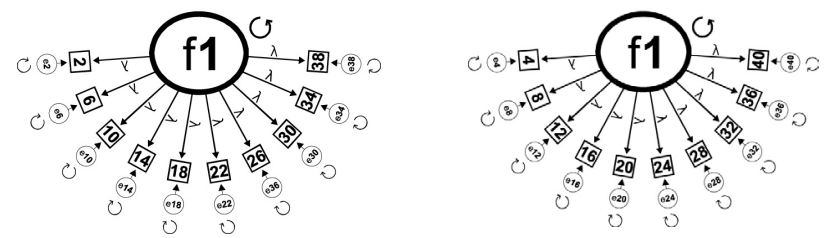

Figura 1: Especificação pictográfica dos modelos que testam, respectivamente, a PAS Mãe e a PAS Pai.

A avaliação da qualidade dos modelos implica a análise dos indicadores de bondade do ajustamento global (cf. Quadro 4). O valor do teste de qui-quadrado para o ajustamento da PAS Mãe e da PAS Pai revela-se adequado, muito embora devido ao número de sujeitos ( $n=1276$ e $n=1234$ ) relativamente aos quais foram obtidas respostas válidas o nível de significância seja muito baixo. No sentido de ultrapassar este inconveniente foi necessário considerar em simultâneo a informação de outras medidas como o CFI (.92: PAS Mãe; .95: PAS Pai) e TLI (.98: PAS Mãe; .99: PAS Pai) que indicam um modelo fortemente ajustado. Calculou-se, ainda, o RMSEA (.10 em ambos) cujo valor acima do limite desejado pode ser interpretado como 
uma aproximação tangencial à matriz de covariância da população (SchermellehEngel, Moosbrugger \& Müller, 2003). Este valor encontrado para o RMESA parece indicar a existência de uma discrepância entre as matrizes originais consideradas para a população e as matrizes obtidas considerando os dados observados, tendo em apreciação as especificações que se realizaram na definição dos modelos (Schermelleh-Engel, Moosbrugger \& Müller, 2003). Contudo, será de referir que a interpretação desta medida tem de ser cuidadosa, na medida em que existe pouca informação relativamente ao seu comportamento quando estamos perante dados de natureza categorial (Muthén, 1999 referido por Kim \& Mueller, 2001), como é o caso presente.

Quadro 4: Indicadores da bondade do ajustamento do modelo testado para as variáveis PAS Mãe ( $n=1276)$ e PAS Pai $(n=1234)$.

\begin{tabular}{llllc}
\hline \multicolumn{5}{l}{ Indicadores de ajustamento } \\
Modelos & X2 $(\mathrm{gl})$ & $\mathrm{CFI}$ & $\mathrm{TLI}$ & RMSEA \\
\hline PAS Mãe & $395.82(27)^{* *}$ & .92 & .98 & .10 \\
PAS Pai & $389.56(28)^{* *}$ & .95 & .99 & .10 \\
\hline
\end{tabular}

${ }^{* *} p<.001$

Em seguida, consideram-se os indicadores locais de ajustamento para a PAS Mãe, observando os coeficientes de cada item na variável latente que se pressupõe que estejam a marcar. De um modo global, percebe-se um nível bastante adequado de ajustamento dos itens e que todos os loadings no factor são estatisticamente significativos 5 .

Este modelo revela-se adequado à interpretação da estrutura subjacente à percepção da aceitação da mãe. Os valores de ajustamento corroboram esta sugestão e não são sugeridas modificações do ponto de vista estatístico, isto é, índices de modificação que implicariam uma redução de pelo menos 10 pontos no valor da estatística de qui-quadrado.

Na observação aos coeficientes do modelo estimado para a PAS Pai verifica-se que os valores dos loadings na variável latente são significativamente diferentes de zero.

Uma vez mais o software utilizado não sugeriu modificações à estrutura proposta e apreciação global aos indicadores permite afirmar que se está perante um bom modelo explicativo do construto de percepção da aceitação do pai.

5 Os valores dos indicadores de ajustamento local não serão apresentados para os 4 modelos construídos separadamente. Esta opção justifica-se por se considerar que esta informação sobrecarregaria o texto e que estes, embora diferentes dos gerados no modelo global, não se afastam deste de forma considerável. 


\subsubsection{Percepção da Aceitação dos Amigos e Percepção da Aceitação da Família}

A estrutura factorial avançada para as subescalas da Percepção da Aceitação dos Amigos e da Família considera dois factores, constituídos por seis itens cada um. O primeiro factor engloba os itens que remetem para aspectos de aceitação, possuindo uma orientação positiva. O segundo factor é composto pelas questões que podem ser percepcionadas pelos indivíduos como rejeição, ou seja, itens com orientação negativa. Seguiu-se esta opção na especificação do modelo uma vez que em trabalhos anteriores as Análises em Componentes Principais indicavam essa configuração (Brock et al., 1998; Figueiredo, Maia \& Pinheiro, 2004; Pinheiro, 2003).

Desta forma na especificação pictográfica dos modelos subjacentes à PAS Amigos e à PAS Família observam-se duas variáveis latentes correlacionadas entre si, cada uma das quais marcada por seis variáveis categoriais (cf. Figura 2).
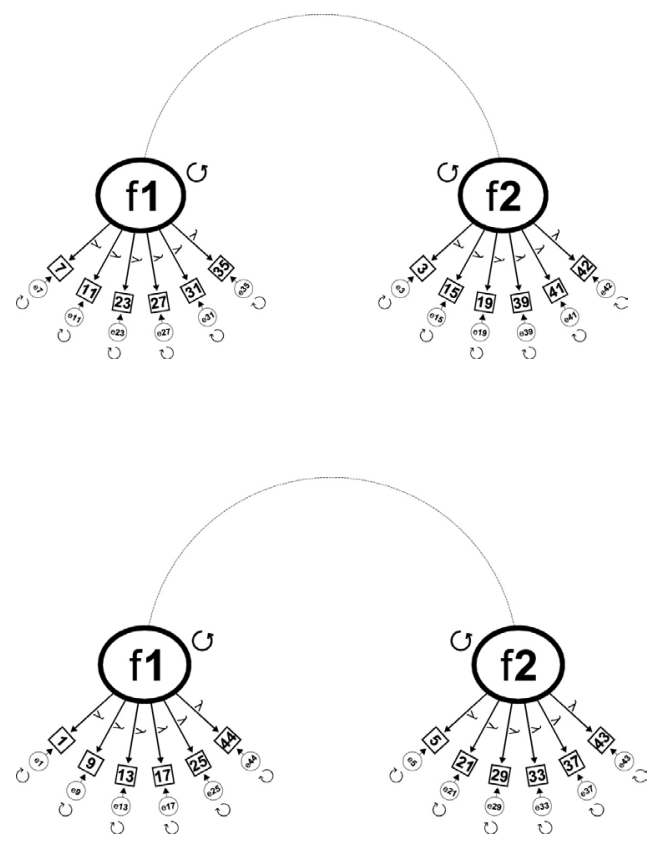

Figura 2: Especificação pictográfica do modelo que testa respectivamente, a PAS Amigos e a PAS Família.

Os indicadores gerais relativos à bondade do ajustamento revelam os modelos propostos como bons quadros de leitura no que respeita aos construtos de percepção da aceitação dos amigos e da família (cf. Quadro 5). Os quatro indicadores que têm sido referenciados neste diagnóstico revelaram-se bastante satisfatórios para a PAS Amigos e um pouco inferiores para a PAS Família, mas igualmente aceitáveis. 
Quadro 5: Indicadores da bondade do ajustamento do modelo testado para as variáveis PAS Amigos ( $n=1290)$ e PAS Família $(n=1287)$.

\begin{tabular}{llccc}
\hline \multicolumn{5}{c}{ Indicadores de ajustamento } \\
Modelos & $\chi^{2}(\mathrm{gl})$ & CFI & TLI & RMSEA \\
\hline PAS Amigos & $334.20(16)^{* *}$ & .95 & .98 & .08 \\
PAS Família & $458.04(40)^{* *}$ & .93 & .98 & .09 \\
\hline
\end{tabular}

** $p<.001$

Quanto ao ajustamento local, os coeficientes calculados para os dois factores, considerados na PAS Amigos, para além de fazerem sentido do ponto de vista substantivo, são estatisticamente significativos.

$\mathrm{Na}$ apreciação global da PAS Amigos verifica-se ainda um coeficiente de correlação de .74 entre os dois factores, o que parece fazer sentido por se tratarem de constructos complementares (aceitação e rejeição).

Em sintese, a estrutura factorial da percepção da aceitação dos amigos, conforme foi considerada aquando da construção do instrumento e das suas variáveis observadas, revela-se bastante adequada. Neste sentido, e em termos do que pode ser a investigação e a intervenção no domínio do ensino superior a PAS Amigos, de forma isolada, apresenta características que reforçam a confiança na sua utilização. Esta conclusão merece ser realçada na medida em que este construto se prevê ser uma cognição transferida para a percepção do relacionamento com os colegas de curso, que são entidades que se revelam de grande importância no sucesso e envolvimento académico (Astin, 1993; Bastos, 2000; Knefelkamp, 1999).

Quanto ao ajustamento local da PAS Família observa-se que todos os loadings são estatisticamente significativos.

O valor de correlação entre os dois factores é de .86 , magnitude que seria de esperar atendendo à natureza das duas variáveis, sendo este valor superior ao reportado por Pinheiro (2003) nos seus estudos.

Em síntese, e numa visão global, assume-se que o modelo proposto para esta subescala oferece um nível razoável de poder na interpretação do construto.

\subsubsection{A Escala de Percepção da Aceitação: estudo global do modelo considerando as quatro subescalas}

A análise da estrutura factorial da PAS num modelo que a compreendesse na sua totalidade e, deste modo, possibilitasse uma visão de conjunto acerca da qualidade do instrumento, é realizada pela primeira vez. Esta análise torna-se concretizável 
pela utilização da Análise Factorial Confirmatória que oferece garantias que os métodos anteriores não ofereciam ${ }^{6}$. Este estudo considerou quatro variáveis latentes de percepção da aceitação, referentes aos amigos, à mãe, ao pai e à família, as mesmas que tinham sido avaliadas de forma isolada, anteriormente. Estas variáveis correlacionadas entre si são marcadas pelos 44 itens que compõem a Escala de Percepção da Aceitação (10 para a subescala da mãe e do pai, 12 para as subescalas dos amigos e da família) (cf. Figura 3).

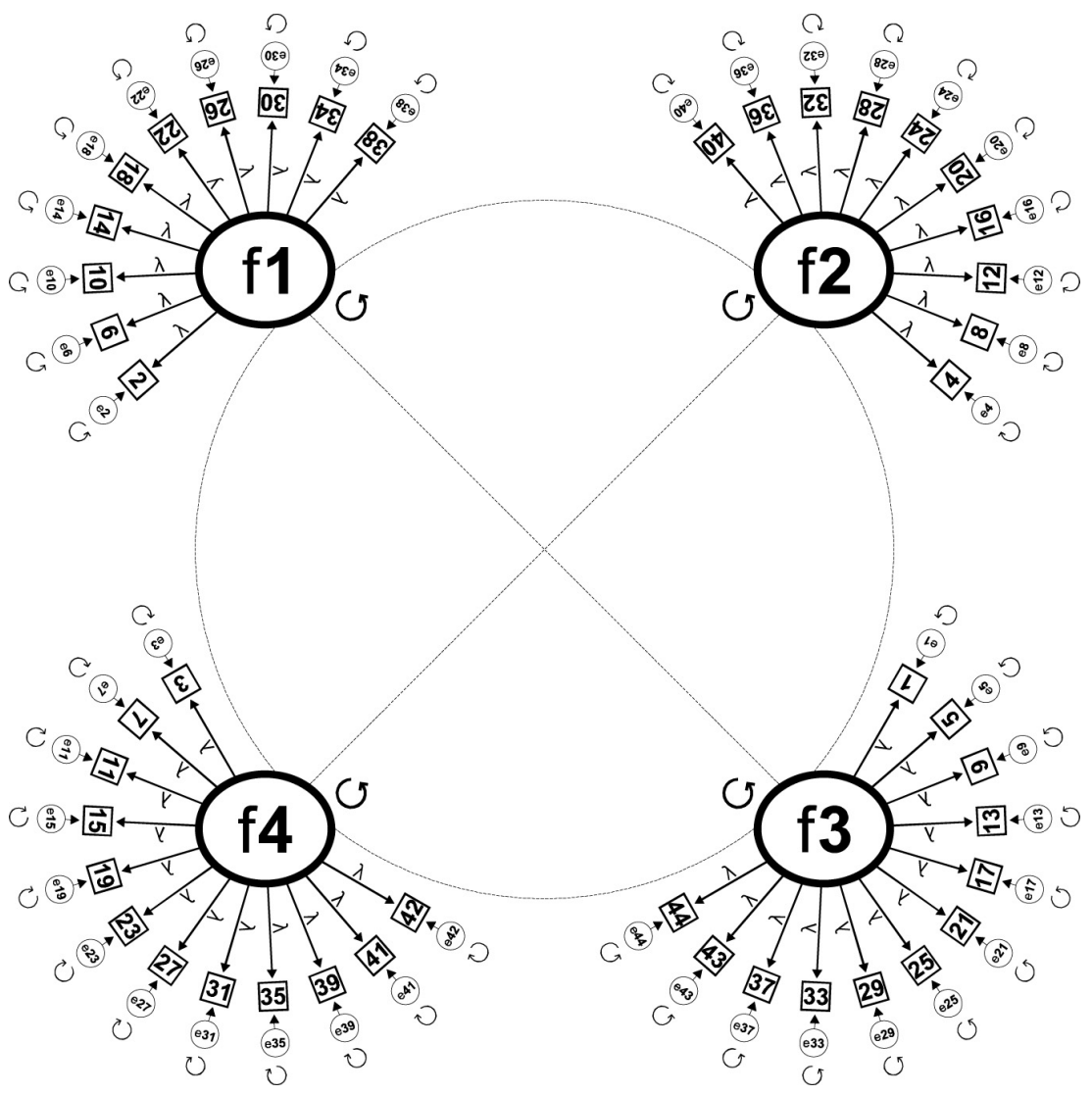

Figura 3: Especificação pictográfica do modelo que testa a PAS.

6 Os estudos da PAS global realizados anteriormente utilizaram Análises em Componentes Principais, que devido ao modo de construção das subescalas, se apresentam limitativas, uma vez que seguindo este procedimento se observa o agrupamento dos itens de conteúdos semelhantes e não os itens referente às entidades em avaliação (mãe, pai, amigos e família). Por outro lado, verifica-se o agrupamento de itens da mãe e do pai com itens referentes à subescala da família. Assim, apenas com um procedimento que permite definir à priori os pressupostos subjacentes à construção deste instrumento seria possível testá-lo. 
Numa primeira análise ao modelo verifica-se que os valores de bondade do ajustamento indicam que se adapta de forma pouco satisfatória aos dados recolhidos (cf. Quadro 7). Porém, tendo sido esta escala escrita de forma simétrica, com itens que expressam conteúdos relativos à aceitação mas apelando também a outros aspectos psicológicos, será de prever que as variâncias-erro de alguns itens estejam correlacionadas, em particular se estes pertencerem às subescalas mãe-pai e família-amigos.

Na tentativa de construir um modelo que melhor interpretasse as quatro variáveis latentes mas que simultaneamente se ajustasse aos dados e às características particulares deste instrumento efectuaram-se 10 modificações ao modelo inicial (cf. Quadro 6).

O assumir destas modificações resultou da reflexão acerca de dois aspectos fundamentais: a justificação substantiva com base no quadro interpretativo do construto e os índices de modificação disponibilizados pelo software. Estes últimos caracterizam-se num conjunto de procedimentos e estatísticas que informam sobre as possíveis causas de má especificação do modelo, ou seja, realizam um diagnóstico em termos das estimativas dos parâmetros (magnitude e sinal), valor dos erros padrão, estimativas de parâmetros não considerados no modelo e que podem ser fixados em zero. Deste modo, permitem que se realizem ajustamentos, mas apenas e só se estes forem devidamente sustentados do ponto de vista teórico (Byrne, 1994).

As modificações admitidas na configuração da PAS foram introduzidas separadamente no modelo, gerando assim a cada passo um novo modelo hierarquicamente superior ao anterior, ou seja, contendo todas as especificações do antecedente mais a nova modificação. Em cada um dos passos analisaram-se os valores de ajustamento e os índices de modificação.

Nas modificações 1, 2, 3, 4, 5, 6, 7 e 10 estamos perante itens cujo conteúdo semântico é semelhante, apenas se distinguindo nas entidades a que se referem. A modificação número 8 correlaciona as variâncias-erro de dois itens da mesma subescala, a PAS Amigos, mas o tipo de percepção a que faz apelo parece ser realmente muito similar, apenas escrita em sentidos contrários. Efectivamente, quando alguém se sente à margem das coisas pode exprimir o sentimento de que não sabe se os outros gostam realmente de si, ou pelo contrário, quando se está por dentro e se sente uma parte importante do grupo de amigos não se questionam se os amigos gostam realmente de si. A modificação a que foi atribuida o número 9 correlaciona a variância de erro associada ao ser castigado pelos pais ou apenas pela figura paterna (cf. Quadro 6). 
Quadro 6: Modificações realizadas ao modelo inicial da PAS.

\begin{tabular}{|c|c|c|c|}
\hline Modificações & Itens & Subescala & Conteúdo \\
\hline \multirow{2}{*}{$\mathrm{m} 1$} & 22 & PAS Mãe & $\begin{array}{l}\text { Sinto-me bem, sendo “apenas eu proprio/a”, } \\
\text { quando estou com a minha mãe. }\end{array}$ \\
\hline & 16 & PAS Pai & $\begin{array}{l}\text { Sinto-me bem, sendo "apenas eu proprio/a”, } \\
\text { quando estou com o meu pai. }\end{array}$ \\
\hline \multirow{2}{*}{$\mathrm{m} 2$} & 40 & PAS Pai & $\begin{array}{l}\text { O meu pai acredita que aquilo que faço é o melhor } \\
\text { para mim. }\end{array}$ \\
\hline & 38 & PAS Mãe & $\begin{array}{l}\text { A minha mãe acredita que aquilo que faço é o } \\
\text { melhor para mim. }\end{array}$ \\
\hline \multirow{2}{*}{ m3 } & 30 & PAS Mãe & A minha mãe castigou-me vezes demais. \\
\hline & 4 & PAS Pai & O meu pai castigou-me vezes demais. \\
\hline \multirow{2}{*}{ m4 } & 28 & PAS Pai & $\begin{array}{l}\text { A minha mãe, apesar do que eu possa dizer ou } \\
\text { fazer, ama-me e preocupa-se comigo. }\end{array}$ \\
\hline & 14 & PAS Mãe & $\begin{array}{l}\text { A minha mãe, apesar do que eu possa dizer ou } \\
\text { fazer, ama-me e preocupa-se comigo. }\end{array}$ \\
\hline \multirow{2}{*}{ m5 } & 36 & PAS Pai & $\begin{array}{l}\text { O meu pai teve sempre cuidado para não magoar } \\
\text { os meus sentimentos. }\end{array}$ \\
\hline & 34 & PAS Mãe & $\begin{array}{l}\text { A minha mãe teve sempre cuidado para não ma- } \\
\text { goar os meus sentimentos. }\end{array}$ \\
\hline \multirow{2}{*}{$\mathrm{m} 6$} & 39 & PAS Família & $\begin{array}{l}\text { Muitas vezes, sinto que a minha família não me } \\
\text { compreende. }\end{array}$ \\
\hline & 37 & PAS Amigos & $\begin{array}{l}\text { Muitas vezes, sinto que os meus amigos não me } \\
\text { entendem. }\end{array}$ \\
\hline \multirow{2}{*}{$\mathrm{m} 7$} & 43 & PAS Amigos & $\begin{array}{l}\text { Os meus amigos opuseram-se a um certo número } \\
\text { de coisas que eu fiz. }\end{array}$ \\
\hline & 3 & PAS Família & $\begin{array}{l}\text { Os meus pais opuseram-se a um certo número de } \\
\text { coisas que eu fiz. }\end{array}$ \\
\hline \multirow{2}{*}{$\mathrm{m} 8$} & 21 & PAS Amigos & $\begin{array}{l}\text { Nas relações com os outros, sinto-me muitas vezes } \\
\text { à margem das coisas. }\end{array}$ \\
\hline & 5 & PAS Amigos & $\begin{array}{l}\text { Por vezes, pergunto a mim mesmo se as outras } \\
\text { pessoas gostam de mim. }\end{array}$ \\
\hline \multirow[b]{2}{*}{ m9 } & 4 & PAS Pai & O meu pai castigou-me vezes demais. \\
\hline & 3 & PAS Família & $\begin{array}{l}\text { Os meus pais opuseram-se a um certo número de } \\
\text { coisas que eu fiz. }\end{array}$ \\
\hline \multirow{2}{*}{ m10 } & 41 & PAS Família & $\begin{array}{l}\text { Sinto-me na zona limite (nem dentro nem fora) das } \\
\text { minhas relações familiares. }\end{array}$ \\
\hline & 29 & PAS Amigos & $\begin{array}{l}\text { Sinto-me na zona limite (nem dentro nem fora) do } \\
\text { meu grupo de amigos. }\end{array}$ \\
\hline
\end{tabular}

Na avaliação da relevância das modificações introduzidas atendeu-se fundamentalmente à diferença em termos de qui-quadrado de um modelo mais simples para um modelo mais complexo, sendo assumido um bom ganho no modelo mais complexo quando o valor desta medida for estatisticamente significativo. Estas diferenças de qui-quadrado são calculadas utilizando um procedimento disponível no software Mplus, uma vez que não pode ser realizada fazendo a subtracção directa entre os valores de qui-quadrado e graus de liberdade (gl) obtidos num modelo e os valores do modelo seguinte, como é estratégia habitual no caso dos dados serem contínuos. Este cálculo alternativo terá de ser efectuado sempre 
que se utiliza o estimador WLSMV porque a diferença entre os valores do teste dos modelos não segue uma distribuição de qui-quadrado (Muthén \& Muthén, 2005). Analisaram-se igualmente os outros índices de bondade do ajustamento. Todos os dez passos seguidos até à construção de um modelo final composto pela configuração inicial mais a correlação das 10 variâncias-erro revelaram diferenças de qui-quadrado estatisticamente significativas, assim como ganhos nos três índices de ajustamento referidos (cf. Quadro 10).

Após estes reajustamentos obteve-se um modelo final que se ajusta de forma mais aceitável aos dados. Contudo, o RMSEA (.o8) esta no ponto de corte para se poder considerar o modelo com um grau de aproximação razoável e o valor de CFI (.82) parece relativamente baixo. Contudo, será de lembrar que o CFI é uma medida sensivel ao número de parâmetros a estimar e ao número de efectivos da amostra (Cheung \& Rensvold, 2000). O TLI (.96) aponta no sentido do modelo na sua globalidade ser uma grelha de leitura bastante aceitável (cf. Quadro 7).

Quadro 7: Indicadores da bondade do ajustamento dos modelos testados para a PAS após introdução de modificações e diferença de qui-quadrado ( $n=1226)$.

\begin{tabular}{|c|c|c|c|c|c|}
\hline Modelos & $x^{2}(\mathrm{gl})$ & $\Delta \chi_{2}^{2}(\mathrm{gl})$ & $\mathrm{CFI}$ & TLI & RMSEA \\
\hline Modelo inicial & $2603.39(221)$ & & .75 & .95 & .09 \\
\hline $\mathrm{Ml}+\mathrm{m} 1$ & $2430.08(220)$ & $340.10(1)$ & .77 & .95 & .09 \\
\hline $\mathrm{Ml}+\mathrm{m} 1+\mathrm{m} 2$ & $2255 \cdot 55(220)$ & $478.11(1)$ & .79 & .96 & .09 \\
\hline$M l+m 1+m 2+m_{3}$ & $2147.90(220)$ & $302.09(1)$ & .80 & .96 & .09 \\
\hline $\mathrm{Ml}+\mathrm{m}_{1}+\mathrm{m}_{2}+\mathrm{m}_{3}+\mathrm{m}_{4}$ & $2114.57(220)$ & $158.94(1)$ & .80 & .96 & .08 \\
\hline $\mathrm{Ml}+\mathrm{m}_{1}+\mathrm{m}_{2}+\mathrm{m}_{3}+\mathrm{m}_{4}+\mathrm{m}_{5}$ & $2080.29(220)$ & $156.35(1)$ & .81 & .96 & .08 \\
\hline $\mathrm{Ml}+\mathrm{m}_{1}+\mathrm{m}_{2}+\mathrm{m}_{3}+\mathrm{m}_{4}+\mathrm{m}_{5}+\mathrm{m}_{6}$ & $2047.49(220)$ & $123.16(1)$ & .81 & .96 & .08 \\
\hline $\mathrm{Ml}+\mathrm{m}_{1}+\mathrm{m}_{2}+\mathrm{m}_{3}+\mathrm{m}_{4}+\mathrm{m}_{5}+\mathrm{m}_{6}+\mathrm{m}_{7}$ & $2009.31(220)$ & $87.05(1)$ & .81 & .96 & .08 \\
\hline $\begin{array}{l}\mathrm{Ml}+\mathrm{m}_{1}+\mathrm{m}_{2}+\mathrm{m}_{3}+\mathrm{m}_{4}+\mathrm{m}_{5}+\mathrm{m} 6+\mathrm{m}_{7} \\
+\mathrm{m} 8\end{array}$ & $1992.83(220)$ & $59.65(1)$ & .81 & 96 & .08 \\
\hline $\begin{array}{l}\mathrm{Ml}+\mathrm{m} 1+\mathrm{m}_{2}+\mathrm{m}_{3}+\mathrm{m}_{4}+\mathrm{m}_{5}+\mathrm{m} 6+\mathrm{m}_{7} \\
+\mathrm{m} 8+\mathrm{mg}\end{array}$ & $1996.63(220)$ & $83.78(1)$ & .82 & .96 & .08 \\
\hline $\begin{array}{l}\mathrm{Ml}+\mathrm{m} 1+\mathrm{m} 2+\mathrm{m}_{3}+\mathrm{m} 4+\mathrm{m}_{5}+\mathrm{m} 6+\mathrm{m} 7 \\
+\mathrm{m} 8+\mathrm{m} 9+\mathrm{m} 10 \\
\text { (modelo final) }\end{array}$ & $1939.91(220)$ & $87.85(1)$ & .82 & .96 & .08 \\
\hline
\end{tabular}

Nota: Todos os valores de $x 2$ são estatisticamente significativos.

$\mathrm{Na}$ avaliação do ajustamento local constata-se que todos os coeficientes calculados para o modelo são significativamente diferentes de zero (cf. Quadro 8 e 9). No factor 1, a PAS Mãe, os coeficientes estandardizados variam entre .66 e .82. 0 item que tem maior percentagem de variância explicada pelo factor é o número 18 (A minha mãe nunca me compreendeu verdadeiramente) enquanto o que possui menor percentagem de variância é o 22 (Sinto-me bem, sendo "apenas eu próprio/a", quando estou com a minha mãe). 
Quadro 8: Loading do item no factor, respectivo erro estandardizado e coeficiente estandardizado para as subescalas de percepção da aceitação da mãe (F2) e do pai (F3).

\begin{tabular}{lcccc}
\hline Factor & Item & Coeficiente \pm Erro padrão & $\begin{array}{c}\text { Coeficiente } \\
\text { estandardizado }\end{array}$ & R2 \\
\hline 2 & $1.00^{1}$ & .68 & .46 \\
& 6 & $1.10 \pm .04$ & .75 & .56 \\
& 10 & $1.14 \pm .04$ & .78 & .61 \\
& 14 & $1.10 \pm .04$ & .76 & .57 \\
& 18 & $1.21 \pm .04$ & .82 & .68 \\
& 22 & $.96 \pm .04$ & .66 & .43 \\
& 26 & $1.19 \pm .04$ & .81 & .66 \\
& 30 & $.96 \pm .04$ & .66 & .43 \\
& 34 & $1.06 \pm .04$ & .72 & .52 \\
& 38 & $.98 \pm .04$ & .67 & .44 \\
\hline 4 & $1.00 \pm .00$ & .53 & .28 \\
& 8 & $1.43 \pm .07$ & .76 & .57 \\
& 12 & $1.46 \pm .07$ & .77 & .60 \\
& 16 & $1.24 \pm .07$ & .65 & .43 \\
& 20 & $1.48 \pm .08$ & .78 & .61 \\
& 24 & $1.69 \pm .07$ & .90 & .80 \\
& 28 & $1.52 \pm .07$ & .80 & .64 \\
32 & $1.42 \pm .07$ & .75 & .57 \\
& 36 & $1.39 \pm .07$ & .74 & .54 \\
& 40 & $1.33 \pm .07$ & .50 \\
\hline
\end{tabular}

Quanto à PAS Pai (F2) observa-se que o item que se destaca de forma particular na marcação desta variável é o 24 (O meu pai tem-me feito sentir que realmente me aceita). O item número 4 ( $O$ meu pai castigou-me vezes demais) foi aquele com o coeficiente estandardizado mais baixo (.53), assim como um valor de $\mathrm{R}^{2}$ reduzido (.28) tanto neste modelo que contempla a totalidade da PAS ou apenas na PAS Pai.

$\mathrm{Na}$ PAS Amigos ( $\mathrm{F}_{3}$ ) os itens 43 (Os meus amigos opuseram-se a um certo número de coisas que eu fiz) e 5 (Por vezes, pergunto a mim mesmo se as outras pessoas gostam de mim) apresentam os coeficientes estandardizados mais baixos, respectivamente .49 e .51. Por outro lado, são os itens 21 (Nas relações com os outros sinto-me muitas vezes à margem das coisas) e 29 (Sinto que estou na zona limite (nem dentro nem fora) do meu grupo de amigos) que revela coeficientes mais expressivos (respectivamente, .73 e .71) (cf. Quadro 9). 
Quanto à PAS Família (F4) os valores dos coeficientes estandardizados variam entre .45 e .77 , correspondendo estes valores aos itens 3 (Os meus pais opuseram-se a um certo número de coisas que fiz) e 23 (A minha familia mostra frequentemente que se preocupa comigo), respectivamente (cf. Quadro 9).

Quadro 9: Loading do item no factor, respectivo erro estandardizado e coeficiente estandardizado para as subescalas de percepção da aceitação dos amigos (F1) e da família (F4).

\begin{tabular}{|c|c|c|c|c|}
\hline Factor & Item & Coeficiente \pm Erro padrão & $\begin{array}{c}\text { Coeficiente } \\
\text { estandardizado }\end{array}$ & $\mathrm{R}_{2}$ \\
\hline \multirow{12}{*}{1} & 1 & 1.00 & .55 & .30 \\
\hline & 5 & $.94 \pm .06$ & .51 & .26 \\
\hline & 9 & $1.25 \pm .06$ & .68 & .47 \\
\hline & 13 & $1.25 \pm .07$ & .68 & .47 \\
\hline & 17 & $1.22 \pm .06$ & .66 & .44 \\
\hline & 21 & $1.33 \pm .07$ & .73 & .53 \\
\hline & 25 & $.61 \pm .06$ & .33 & .11 \\
\hline & 29 & $1.03 \pm .07$ & .71 & .50 \\
\hline & 33 & $1.28 \pm .07$ & .70 & .48 \\
\hline & 37 & $1.19 \pm .06$ & .65 & .42 \\
\hline & 43 & $.90 \pm .07$ & .49 & .24 \\
\hline & 44 & $1.16 \pm .07$ & .63 & .40 \\
\hline \multirow{12}{*}{4} & 3 & $1.00 \pm .00$ & .45 & .21 \\
\hline & 7 & $1.56 \pm .09$ & .72 & .51 \\
\hline & 11 & $1.51 \pm .09$ & .69 & .48 \\
\hline & 15 & $1.43 \pm .08$ & .65 & .43 \\
\hline & 19 & $1.37 \pm .08$ & 63 & .40 \\
\hline & 23 & $1.69 \pm .09$ & .77 & .60 \\
\hline & 27 & $1.59 \pm .09$ & .73 & .53 \\
\hline & 31 & $1.60 \pm .09$ & .73 & .53 \\
\hline & 35 & $.92 \pm .07$ & .42 & .18 \\
\hline & 39 & $1.53 \pm .08$ & .70 & .49 \\
\hline & 41 & $1.45 \pm .08$ & .67 & .44 \\
\hline & 42 & $1.55 \pm .09$ & .71 & .51 \\
\hline
\end{tabular}

Um outro aspecto que sobressai da análise dos valores de $R^{2}$ para todos os itens da PAS é a existência de valores particularmente baixos como é o caso dos observados nos itens 25 e 35 (respectivamente, .11 e .18). Numa análise ao conteúdo destas questões verifica-se que são itens semelhantes, pertencentes às subescalas PAS Amigos (25: Os meus segredos mais intimos confio-os aos meus amigos) e PAS Família (35: Os meus sentimentos mais intimos confio-os à minha família). 
Uma possivel interpretação destes valores poderá residir no facto de ser bastante improvável que os indivíduos considerem contar os seus segredos mais íntimos aos amigos, uma vez que esta expressão verbal parece fazer apelo a um tipo de informações que estes podem não partilhar com ninguém. Ao ler este item o estudante poderá pensar em sentimentos, pensamentos ou comportamentos que encara como inconfessáveis. Para se aumentar os níveis de associação entre a variável e o referido item, no âmbito de uma escala de percepção da aceitação, poderia ser oportuno reformula-lo do seguinte modo: sei que posso desabafar com os meus amigos. Do mesmo modo, os sentimentos e os segredos mais íntimos podem não ser fáceis de revelar a alguém, ou pelo menos que revelar determinados segredos pode não ser parte integrante daquilo que caracteriza a aceitação. Uma proposta de reformulação deste item no contexto do construto que se pretende marcar deveria reforçar o aspecto de que os indivíduos se sentem mais aceites se apesar de não confiarem os seus sentimentos mais íntimos às suas famílias, estas os amam e os valorizam. Uma proposta para esta alteração poderia ser escrita do seguinte modo: se sentir necessidade tenho na minha familia a quem confiar os meus sentimentos mais intimos.

Por outro lado, também os itens 3 (PAS Família) e 43 (PAS Amigos) revelam $\mathrm{R}^{2}$ inferiores a .25 (respectivamente .21 e .24) e mais uma vez ambos compreendem conteúdos idênticos (3: Os meus pais opuseram-se a um certo número de coisas que eu fiz; 43: Os meus amigos opuseram-se a um certo número de coisas que eu fiz). Efectivamente, sabe-se que esta oposição poderá ser encarada como rejeição dependendo fundamentalmente do período de desenvolvimento em que os indivíduos se encontram, porque se para uns a oposição dos pais ou amigos será encarada como uma forma de rejeição, para outros é possível coexistir oposição e aceitação. A própria definição do conceito apela para esta noção, considerando esta percepção como uma "apreciação relativamente estável de que os outros se preocupam connosco e nos valorizam e que esse cuidado não é dependente de por vezes as nossas atitudes e acções serem diferentes das habituais" (Brock et al., 1998, p. 6). Neste sentido, uma sugestão seria substituir a expressão "opuseram-se a um certo número de coisas que fiz" por "opõem-se de um modo geral aquilo que faço".

Os coeficientes de correlação entre as quatro variáveis latentes situam entre .44 (PAS Pai e PAS Amigos) e .86 (PAS Mãe e PAS Família) (cf. Quadro 10). Estes resultados são semelhantes aos de Pinheiro (2003) no cálculo da correlação de Pearson entre os totais das subescalas que variaram entre .31 (PAS Pai e PAS Amigos) e .79 (PAS Mãe e PAS Família).

Será interessante notar que a subescala de percepção da aceitação dos amigos revela as correlações mais baixas, parecendo fazer sentido quando se espera que a natureza das relações e o tipo de aceitação que se possui com o grupo de 
amigos seja diferente da estabelecida com as figuras parentais e com a própria família (Fuligni \& Eccles, 1993). A correlação mais elevada com a PAS Família poderá ser facilmente compreendida pela semelhança na estrutura semântica das duas subescalas.

Outro aspecto merecedor de atenção é a forte associação entre a PAS Mãe e a PAS Família, parecendo indicar que os estudantes estarão a recorrer à representação da sua relação com a mãe, e da aceitação esperada por parte desta, para responder aos 12 itens relativos à família.

Quadro 10: Coeficiente de correlação entre as quatro subescalas da PAS.

\begin{tabular}{lcccc}
\hline & PAS Mãe & PAS Pai & PAS Amigos & PAS Família \\
\hline PAS Mãe & 1.00 & & & \\
PAS Pai & .62 & 1.00 & & \\
PAS Amigos & .49 & .44 & 1.00 & \\
PAS Família & .86 & .78 & .58 & 1.00 \\
\hline
\end{tabular}

\subsubsection{Sintese geral dos resultados}

As duas propostas de análise à configuração da Escala de Percepção da Aceitação revelaram valores, de um modo geral, adequados em cada um dos modelos submetidos a teste. Quando foram testados os quatro modelos de forma independente, foi possivel observar a confirmação das estruturas encontradas nas Análises em Componentes Principais efectuadas em momentos anteriores, com a enorme vantagem de que a composição dos factores é confirmada, tal como os autores originais a tinham concebido, com itens de aceitação e rejeição a marcar variáveis latentes nas subescalas dos amigos e da família e com apenas um factor para as da mãe e do pai.

A avaliação da PAS na sua globalidade, apesar de revelar níveis de ajustamento inferiores aos verificados para os quatro modelos apresenta a enorme vantagem de submeter a teste o instrumento tal como este é apresentado aos indivíduos. Deste modo, torna-se possível verificar que as quatro variáveis latentes, com os respectivos itens que as marcam, se encontram correlacionadas e que esta configuração pode ser uma interpretação adequada dos construtos. Por outro lado, os valores das medidas de ajustamento, embora inferiores ao desejado, são bastante razoáveis tendo em conta a complexidade da matriz colocada em prova. Será mesmo de esperar que esta complexidade torne a avaliação do modelo menos evidente. Não esquecendo que terá de ser da responsabilidade de todos os intervenientes nas instituições de ensino superior o apoio e o sucesso dos estudantes ao longo 
das diferentes etapas por que passam no decorrer dos anos de frequência deste nível de ensino é da competência dos serviços de aconselhamento, ou dos gabinetes de apoio ao estudante, tal como referem Steele e McDonald (2000) fornecer à heterogeneidade desta população as ferramentas necessários ao êxito nesta etapa. Para tal, os instrumentos que deverão ter ao seu dispor devem apresentar qualidades fundamentalmente ao nível da sua validade, o que no caso da Escala de Percepção da Aceitação parece ser razoavelmente conseguido. Esta conclusão é retirada uma vez que se pode confirmar, com um nível satisfatório de confiança, a configuração factorial subjacente à interpretação do construto na matriz de dados recolhida, tendo este aspecto sido verificado numa amostra alargada e relativamente heterogénea de estudantes universitários.

\section{Referências bibliográficas}

Astin, A. (1993). What matters in college? Four critical years revisited. San Francisco: Jossey-Bass Publishers.

Bastos, A. (2000). O desenvolvimento psicológico de estudantes do Ensino Superior. O fim da adolescência ou uma transição desenvolvimental? In T. Medeiros \& A. Serpa (orgs.), Adolescência: abordagens, investigações e contextos de desenvolvimento (pp. 274-300). Açores: direcção Regional de Educação.

Brock, D., Sarason, I., Sanghvi, H. \& Gurung, R. (1998). The perceived acceptance scale: development and validation. Journal of Social and Personal Relationships, 15(1), 5-21.

Brown, T. (2006). Confirmatory factor analysis for applied research. New York: The Guilford Press.

Byrne, B. (1994). Structural Equation modeling with EQS and EQS/Windows: Basic concepts, applications and programming. Thousand Oaks: Sage Publications.

Cheung, G. \& Rensvold, R. (2000). Testing measurement invariance using critical values of Fit Indices: a Monte Carlo study. Research Methods Forum, 5, from http:// www. aom.pace.edu/rmd/cheung_files/cheung.htm.

Cronbach, L. J. (1984). Essentials of psychological testing. New York: Harper \& Row.

DeVellis, R. (2003). Scale development: theory and applications ( $2^{\mathrm{a}}$ ed.). Thousand Oaks: Sage Publications.

Figueiredo, C. (2005). Percepção da aceitação: estudo de validade de uma escala em contexto universitário (dissertação de mestrado). Coimbra: Faculdade de Psicologia e de Ciências da Educação da Universidade de Coimbra.

Figueiredo, C.C., Maia, J.A. \& Pinheiro, M. R. (2005). A Percepção da Aceitação dos Amigos: estudo da invariância de género de uma escala de avaliação psicológica para estudantes do ensino superior. In C. Machado, L. Almeida, M. Guisande, M. Gonçalves \& V. Ramalho (orgs.), Avaliação Psicológica: Formas e Contextos (Vol. XI) (pp. 483-489). Braga: Psiquilibrios.

Figueiredo, C., Maia, A. \& Pinheiro, M.R. (2004). Percepção da aceitação:dados preliminares de um estudo de validação com estudantes da Universidade de Coimbra. In C. Machado, L. Almeida, M. Gonçalves \& V. Ramalho (orgs.), Avaliação psicológica: Formas e Contextos (X) (pp. 182-188). Braga: Psiquilíbrios. 
Flora, D. \& Curran, P. (2004). An empirical evaluation of alternative methods of estimation for confirmatory factor analysis with ordinal data. Psychological Methods, 9 (4), 466-491.

Fuligni, A. \& Eccles,J. (1993). Perceived parent-child relationships and early adolescents`orientation toward peers. Developmental Psychology, 29(4), 622-632.

Kim, K. \& Mueller, D. (2001). To balance or not to balance: confirmatory factor analysis of the affect-balance scale. Journal of Happiness Studies, 2, 289-306.

Knefelkamp, L. (1999). Introduction. In W. Perry, Forms of ethical and intellectual development in the college years: a scheme (pp. xi-xxxviii). San Francisco: Jossey-Bass.

Maia, J. A. (1996). Um discurso metodológico em torno da validade de construto: posição de um Lisrealita, in L. S. Almeida, S. Araújo, M. M. Gonçalves, C. Machado \& M. R. Simões (eds.), Avaliação psicológica:formas e contextos (IV) (pp. 43-60). Braga: APPORT.

Muthén, B. (1992). Latent variable modeling in epidemiology. Alcohol Health \& Research World, 16(4), 286-292.

Muthén, L. \& Muthén, B. (2005). Mplus user’s guide : statistical analysis with latent variables. Los Angeles: Muthén \& Muthén.

Nunnaly, J. (1978). Psychometric theory. New York: Harper and Row.

Pasquali, L. (2003). Psicometria: teoria dos testes na psicologia e na educação. Petropolis: Editora Vozes.

Pinheiro, M. R. (2003). Uma época especial: Suporte social e vivências académicas na transição e adaptação ao ensino superior. Dissertação de doutoramento não publicada. Coimbra: Faculdade de Psicologia e de Ciências da Educação da Universidade de Coimbra.

Pinheiro, M. R. \& Ferreira, J. A. (2001, Outubro). Avaliação do suporte social em contexto de ensino superior. Comunicação apresentada no V Seminário de Investigação e Intervenção Psicológica no Ensino Superior, Viana do Castelo.

Sarason, B., Pierce, I. \& Sarason, G. (1990). Social support: The sense of acceptance and the role of relationships. In B. Sarason, I. Sarason e G. Pierce (eds.), Social Suport: An Interactional view (pp. 97-128). New York: John Wiley \& Sons.

Steele, G. \& McDonald, M. (2000). Advising students in transitions. In V. Gordon, W. Habley \& Associates (eds.), Academic advising: a comprehensive handbook (pp. 144-161). San-Francisco: Jossey-Bass.

Schermelleh-Engel, K., Moosbrugger, H. \& Müller, H. (2003). Evaluating the fit of structural equation models: tests of significance and descriptive goodness-of-fit measures. Methods of Psychological Research Online, 8(2), 23-74.

Thompson, B. (2003). Understanding reliability and coefficient alpha, really. In B. Thompson (ed.), Score reliability: contemporary thinking on reliability issues (pp. 3-30). Thousand Oaks: Sage Publications.

Turner, A., Sarason, I. \& Sarason, B. (2001). Exploring the link between parental acceptance and young adult adjustment. Cognitive Therapy and Research, 25(2), 185-199. 


\section{Assessement of family and friends perceived acceptance: contribution for the factorial validity of the Perceived Aceptance Scale (PAS)}

The Perceived Acceptance Scale - PAS (Brock, Sarason, Sanghvi \& Gurung, 1998; portuguesese version of Pinheiro \& Ferreira, 2001) measure, within relationships with father, mother, friends and family, the self appraisal that others care and value us, independently of our occasional attitudes and actions.

Trying to contribute to the process of transcultural validation of PAS in this study, with a college students sample, are presented and discussed the confirmatory factorial analysis results, with a appropriated estimator for dealing with ordinal data. We concluded that the proposed models reproduce adequately the structure of each subscale and the all scale. In local adjustment analysis we verify that the $R^{2}$ values that each factor extracts from each item are situated between .11 and .80 , requiring a carful interpretation of the semantic quality of some of them. There are suggesting changes.

KEY-WORDS: Social support, Psychometry, Percived acceptance, Factorial Confirmatory analysis

\section{Évaluation de la perception de l'acceptation de la famille et des amis : contributions pour la validité de la structure factorielle de L'Echelle de Perception de L'Acceptation (PAS)}

L'Echelle de Perception de L'Acceptation (Brock, Sarason, \& Gurung Sanghvi, 1998; version portugaise de Pinheiro et Ferreira, 2001) mesure, dans le cadre des relations avec le père, la mère, la famille et les amis, l'appréciation personnelle du fait que les autres s'inquiètent avec nous et nous valorisent indépendamment de nos attitudes et actions spécifiques.

Pour contribuer au processus de validation transculturelle de la PAS dans cette étude, à partir d'un échantillon d'étudiants universitaires, sont présentés et discutés les résultats de l'analyse factorielle confirmatoire en utilisant un estimateur approprié pour traiter les données ordinales.

On conclut que les modèles proposés reproduisent de manière adéquate la structure de chaque sous-échelle et l'ampleur dans son ensemble. Dans l'analyse de l'ajustement local, on constate que les valeurs de R2 pour chaque facteur extrait par item se situent entre .11 et .80, ce qui suggère une interprétation attentive de la qualité sémantique de certains d'entre eux. II y a une recommandation des respectives modifications.

MOTS-CLÉS: Support social, Psychométrie, Perception de l'acceptation, Analyse factorielle confirmatoire 